\title{
From Discrete Time Quantum Walk to Continuous Time Quantum Walk in Limit Distribution
}

\author{
Yutaka Shikano ${ }^{1,2} *$ \\ ${ }^{1}$ Institute for Molecular Science, Okazaki, Aichi, Japan. \\ ${ }^{2}$ Department of Physics, Tokyo Institute of Technology, Tokyo, Japan.
}

July 15, 2013

\begin{abstract}
The discrete time quantum walk defined as a quantum-mechanical analogue of the discrete time random walk have recently been attracted from various and interdisciplinary fields. In this review, the weak limit theorem, that is, the asymptotic behavior, of the one-dimensional discrete time quantum walk is analytically shown. From the limit distribution of the discrete time quantum walk, the discrete time quantum walk can be taken as the quantum dynamical simulator of some physical systems.
\end{abstract}

\section{Contents}

1 Introduction 2

2 Review of Discrete Time Quantum Walk 2

3 Limit Distribution of Discrete Time Quantum Walk 7

4 Connection to Dirac Equation $\quad 10$

5 Connection to Continuous Time Quantum Walk 11

5.1 Review of continuous time quantum walk . . . . . . . . . . 11

5.2 Final-time dependent random walk and lazy random walk . . . . 13

5.3 Final-time dependent discrete time quantum walk . . . . . . 15

6 One-dimensional Discrete Time Quantum Walk as Quantum Simulator

*email: yshikano@ims.ac.jp 


\section{Introduction}

A discrete time quantum walk is defined as the quantum-mechanical analogue of the discrete time random walk but is not the quantization of the random walk. Since this description is stroboscopic and unitary, this can be taken as a simple quantum Turing machine. Therefore, there have recently been several theoretical proposals on quantum information processing and many experimental demonstrations in some physical systems. On the other hand, the discrete time quantum walk also attracts mathematicians to better understand the stochastic process. The aim of this review is to connect the mathematical treatments and physical implementations and meanings of the discrete time quantum walk.

First of all, we give a rough explanation of the discrete time quantum walk. As the random walker, which has information of the position, moves to the left or to the right site depending on the result of a coin flip, a quantum walker and a quantum coin are defined as a position quantum state and a two-level quantum state, which is called a qubit, respectively. It is noted that this formulation is different from the quantization of the discrete time random walk. One step operations of the discrete time random and quantum walks are summarized in Fig. 11 Furthermore, a quantum coin flip and a shift should be unitary operations because these processes are subject to quantum mechanics. It is emphasized that the coin flip is replaced by the one-qubit operation and the shift operation keeps the quantum coherence between the position and the coin summarized as a quantum circuit representation in Fig. 2, This mathematical definition will be stated in Sec. 2 ,

As the mathematical motivation, for the asymptotic behaviors, how different between the quantum and classical random walk has been not yet understood in the discrete and continuous cases. While the discrete time quantum walk is experimentally realized under the motivations to realize the primitive of quantum computation, see for more details in Sec. 2, we have not yet understood how robust the discrete time quantum walk is under the influence of the noise. In this review, we analytically derive the limit distribution of the several discrete time quantum walks in the one-dimensional system. We show that the discrete time quantum walk can simulate various quantum dynamics.

\section{Review of Discrete Time Quantum Walk}

Throughout this review, we focus on a one-dimensional discrete time quantum walk (DTQW) with two-dimensional coins. The DTQW is defined as a quantum-mechanical analogue of the classical random walk. The Hilbert space of the system is a tensor product $\mathcal{H}_{p} \otimes \mathcal{H}_{c}$, where $\mathcal{H}_{p}$ is the position space of a quantum walker spanned by the complete orthonormal basis $\{|n\rangle\}(n \in \mathbb{Z})$ and $\mathcal{H}_{c}$ is the coin Hilbert space spanned by the two orthonormal states $|L\rangle=(1,0)^{\mathbf{T}}$ 


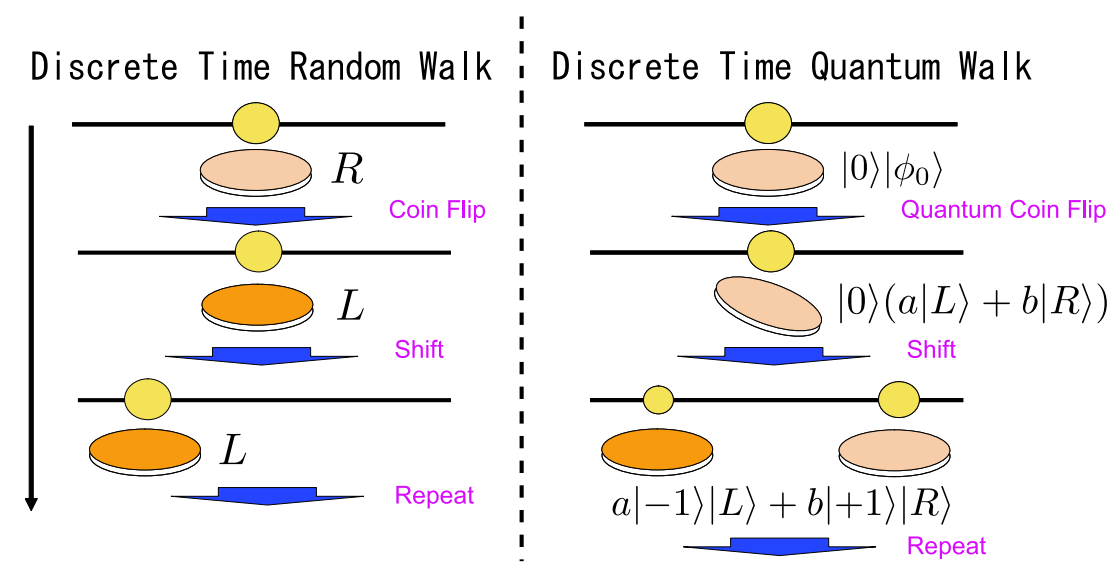

Figure 1: One step of the discrete time random walk v.s. quantum walk. The initial state $|0\rangle\left|\phi_{0}\right\rangle$ expresses that the quantum walker has the quantum coin as $\left|\phi_{0}\right\rangle$ at the origin $|0\rangle$. The coin space is spanned by the two orthogonal states denoted as $|L\rangle$ and $|R\rangle$.

and $|R\rangle=(0,1)^{\mathbf{T}}$. A one-step dynamics is described by a unitary operator $U_{t}=W C_{t}$ with

$$
\begin{aligned}
C_{t} & :=\sum_{n}\left[( a _ { n , t } | n , L \rangle + c _ { n , t } | n , R \rangle ) \left\langlen, L\left|+\left(d_{n, t}|n, R\rangle+b_{n, t}|n, L\rangle\right)\langle n, R|\right],\right.\right. \\
& =\sum_{n}\left[|n\rangle\langle n| \otimes\left(\begin{array}{ll}
a_{n, t} & b_{n, t} \\
c_{n, t} & d_{n, t}
\end{array}\right)\right] \\
& =\sum_{n}\left[|n\rangle\langle n| \otimes \hat{C}_{n, t}\right] \\
W & :=\sum_{n}(|n-1, L\rangle\langle n, L|+| n+1, R\rangle\langle n, R|),
\end{aligned}
$$

where $|n, \xi\rangle=:|n\rangle \otimes|\xi\rangle \in \mathcal{H}_{p} \otimes \mathcal{H}_{c}(\xi=L, R)$ and the coefficients at each position satisfy the following relations: $\left|a_{n, t}\right|^{2}+\left|c_{n, t}\right|^{2}=1, a_{n, t} \bar{b}_{n, t}+c_{n, t} \bar{d}_{n, t}=0, c_{n, t}=$ $-\Delta_{n, t} \bar{b}_{n, t}, d_{n, t}=\Delta_{n, t} \bar{a}_{n, t}$, where $\Delta_{n, t}=a_{n, t} d_{n, t}-b_{n, t} c_{n, t}$ with $\left|\Delta_{n, t}\right|=1$ for any $t$. Two operators $C_{t}$ and $W$ are called coin and shift operators, respectively. The probability distribution at the position $n$ at the $t$ th step is then defined by

$$
\operatorname{Pr}[n ; t]=\sum_{\xi \in\{L, R\}}\left|\left\langle n, \xi\left|\prod_{t} U_{t}\right| 0, \phi_{0}\right\rangle\right|^{2} .
$$

Mathematically speaking, the position of the DTQW at $t$ th step is a random variable denoted as $X_{t}$. It is remarked that a time-independent and homogeneous version of this DTQW was first introduced in Ref. 4. Throughout this 


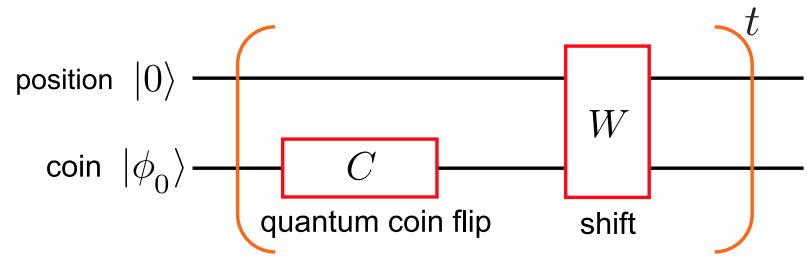

Figure 2: Quantum circuit representation of the discrete time quantum walk. The quantum coin flip and the shift are denoted as $C$ and $W$, respectively. The probability distribution at $t$ th step is given by the position measurement after the partial trace over the quantum coin.

review, we only consider the cases of the time-independent and homogeneous coin, the time-dependent and homogeneous coin, the time-independent and inhomogeneous coin. Therefore, the quantum coin is often described as $\hat{C}_{n, t}=C$ or $\hat{C}_{n, t}=\hat{C}_{n}$ in this review. As a simple example, we show the dynamics of the DTQW with the Hadamard coin given by

$$
\hat{C}_{n, t}=\frac{1}{\sqrt{2}}\left(\begin{array}{cc}
1 & 1 \\
1 & -1
\end{array}\right)
$$

by the third step in the case of the initial coin state $\left|\phi_{0}\right\rangle=|L\rangle$ illustrated in Fig. 3 and at 1000th step in the case of the initial state $\left|\phi_{0}\right\rangle=(|L\rangle+i|R\rangle) / \sqrt{2}$ illustrated in Fig. 4

Historically speaking, the concept of the DTQW was introduced in the independent seminal works in mathematics, physics, and information science. As far as the author knows, Gudder first introduced its concept in his book 25]. While his motivation was to define the stochastic process in quantum probability, he did not mathematically clearly defined the concept in his book. Note that, curiously, he introduced the three step dynamics of the DTQW as the candidate of the stochastic process in quantum probability. However, this is an interesting open question. Second, Aharonov et al. introduced a slightly different version of the DTQW as quantum random walk 2. Their quantum walk is defined as follows. While one-step dynamics is identical to one of the DTQW, in their introduced quantum walk, for every step, the coin state has to be replaced by the initial one. This process is a non-unitary process. While they introduced this as the quantum-mechanical analogue of the classical random walk, their ultimate aim is to construct a process to extract the weak value. Since for every step, the same coin is used, we can extract the weak value of the spin component, for example, $\left\langle\sigma_{z}\right\rangle_{w}$ as the difference of the shift of the position of a quantum particle. This situation mimics the Stern-Gerlach gedankenexperiment. Finally, from the viewpoint of information science, Meyer tried to construct a quantum-mechanical analogue of the cellular automaton to describe dynamics of the one-dimensional quantum gas [57. He showed no-go lemma that keeping the unitarity, a quantum-mechanical analogue of the random walk 


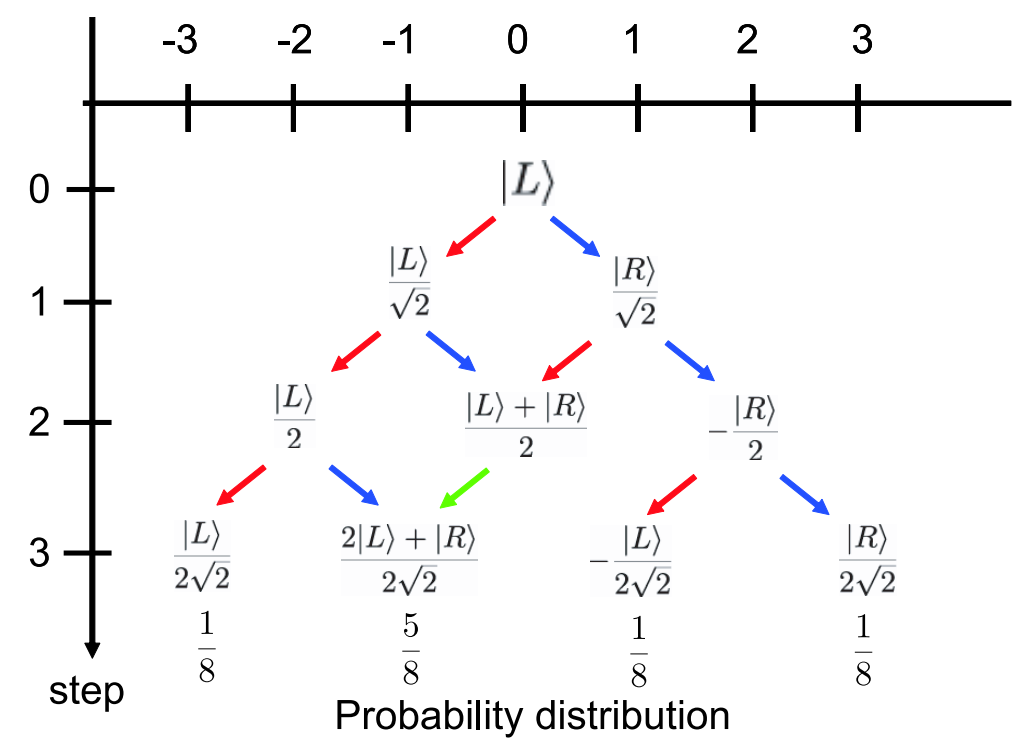

Figure 3: Dynamics of the DTQW with the Hadamard coin (4) by the third step. The coin state is expressed at each position. The initial coin state is $|L\rangle$ at the origin. At the third step, the quantum interference occurred at the origin to be indicated at the green arrow. Therefore, the DTQW does not have the spatial symmetry like the classical random walk. This is dependent of the coin operator and the initial coin state.

cannot be described by a single-component complex-valued function; physically speaking, this is the wavefunction without the spin component.

Since the DTQW is defined as the quantum-mechanical analogue of the classical random walk, the DTQW has many applications to some systems like the classical random walk as the theoretical studies. For examples, in quantum information science, the DTQW is used as the quantum-speedup algorithms $[5,8,52,53$. . The Grover algorithm, which is the spatially search algorithm, can be taken as the tool of the DTQW 68. Furthermore, the LiebRobinson bound, which is the upper bound of the quantum-speedup algorithm with the energy gap, is related to the DTQW in Ref. 111. The DTQW on a graph is a primitive of universal quantum computation [50, 1. See more details in the book [79] and the paper [80]. Furthermore, the entanglement of the DTQW has been analyzed in Refs. [1,9, 49, 54 for the coin and space state of a single particle and in Refs. 62,72] for multi particles. Also, the DTQW is used as the quantum simulator on the quantum phase transition from the Mott insulator to the super-fluid phase, which means the long-range coherence [12, the LandauZehner transition [59], and the classification of the topological phase [37. Recently, there are the theoretical progresses on the multi-particle DTQW for the

\footnotetext{
${ }^{1}$ In the case of the continuous time quantum walk, this is also satisfied [13.
} 


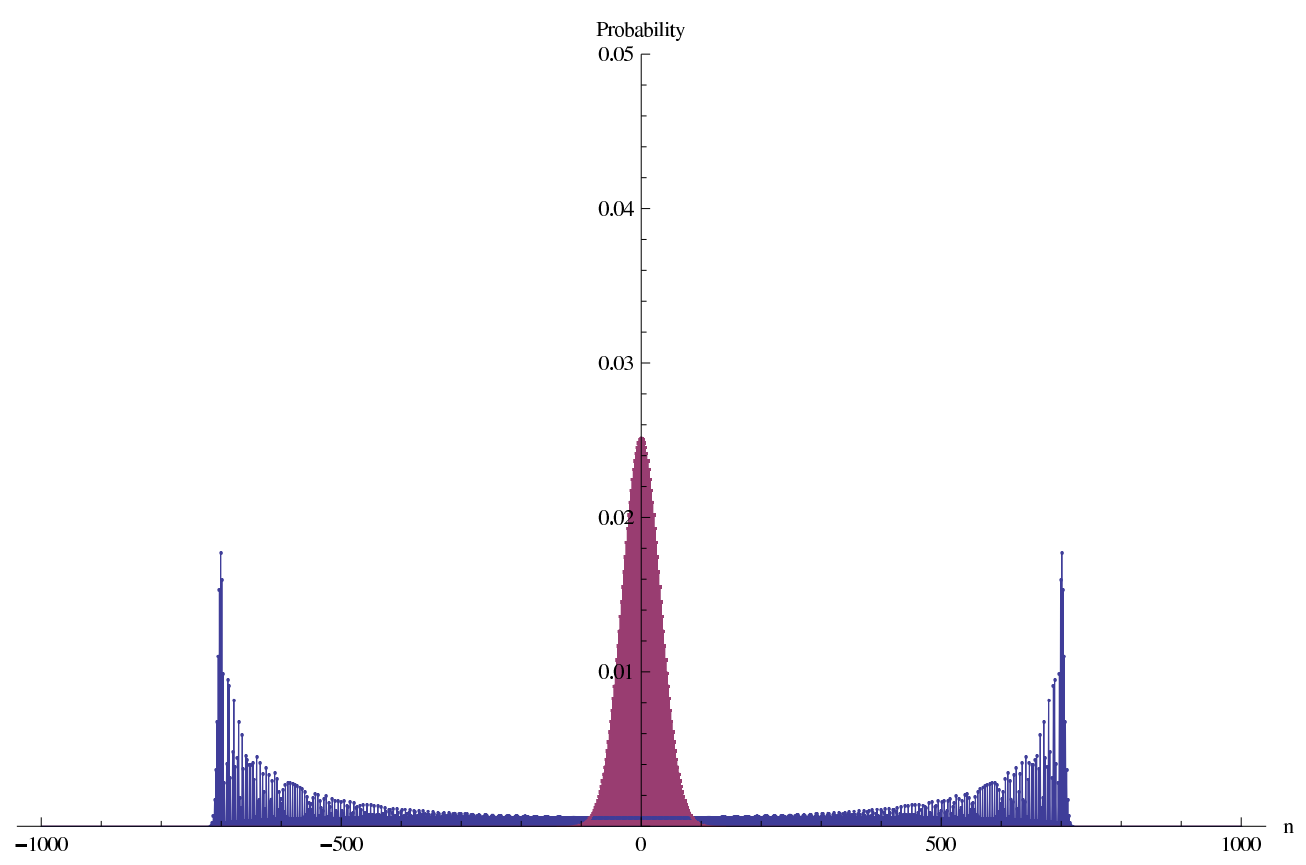

Figure 4: Probability distribution of the DTQW with the Hadamard coin (4) at the 1000th step with the initial coin state $(|L\rangle+i|R\rangle) / \sqrt{2}$.

non-interacting case [23,55] and the interacting one [3, 46]. On the physical properties on the DTQW, see the review papers [33 35]. As the mathematical aspects, there are many analytical studies on the asymptotic behaviors on the DTQW to see more details in the book 41. Almost all classes of the DTQW except for a one-dimensional one with a two-dimensional coin [24, 38, 39, have the aspect of the localization defined that the limit distribution of the DTQW divided by some power of the time variable has the probability density given by the Dirac delta function. In the one dimensional DTQW, the localization was shown in the models with a three-dimensional coin [29], a four-dimensional coin [28, a two-dimensional coin with memory [56, a two-dimensional coin in a random environment [31, two-dimensional coins [48, an spatially incommensurate coin [70] and a time-dependent two-dimensional coin on the Fibonacci quantum walk $[63$ and a random coin $[30,58$. On the other hand, the nature of the localization in the two-dimensional DTQW has been studied numerically [51,78 and analytically 27, 81. The DTQW on the Cayley tree with a multi-state coin was also studied [15]16. Furthermore, the recurrence properties of the DTQW, i.e., the decay rate of the probability at the origin, were studied in Refs. [10,73,75, 82, 83. Recently, the localization property of the DTQW with the inhomogeneous two-dimensional coin has been analytically shown in the model in which the coin at the origin is different from the rest 43,44] and in the model of the periodically inhomogeneous coins 47 . 
It is curiously pointed out that the DTQW has not been found in natural phenomena while the classical random walk was used as the model of the geometrical structure of the complex polymer, "for more details see the book [21]. However, the DTQW has been experimentally realized. In the system of the molecule with the nuclear magnetic resonance, there is the experimental realization by third step [65. In the system of the ion trap, there are theoretical proposals and their experimental realization with ${ }^{40} \mathrm{Ca}^{+}$ion 84 to be motivated by the quantum simulator of the Dirac equation 22. In the system of the optical lattice, there are theoretical proposals and their experimental realizations [18,32. Furthermore, there are experimental demonstration of the DTQW by three step [67, of the DTQW with decoherence [7, and on the topological phase [36] in the optical system 2 .

\section{Limit Distribution of Discrete Time Quantum Walk}

In the following, we focus on the asymptotic behavior of the DTQW. This is because the asymptotic behavior is easily computed and is the most fundamental property of the stochastic process like taking the thermodynamic limit in statistical mechanics. Studying the asymptotic behaviors of the stochastic process is similar to study the connection between the microscopic and macroscopic descriptions. From the viewpoint of probability theory, we obtain the weak limit theorem $a$ la the central limit theorem of the classical random walk (A.1).

Theorem 3.1 (Konno [38,39]). Let $X_{t}^{(D)}$ be the random variable of the homogeneous and time-independent DTQW at time $t$ with the coin operator

$$
C=\left(\begin{array}{ll}
a & b \\
c & d
\end{array}\right)
$$

and the initial coin state

$$
\left|\phi_{0}\right\rangle=q_{L}|L\rangle+q_{R}|R\rangle
$$

with $q_{L}, q_{R} \in \mathbb{C}$. Therefore, we obtain

$$
\frac{X_{t}^{(D)}}{t} \Rightarrow K(|a|)
$$

which $\Rightarrow$ means the weak convergence 3 . Here, $K(r)$ has the probability density function $f(x ; r)$ with a parameter $r \in(0,1)$ :

$$
f(x ; r)=\frac{\sqrt{1-r^{2}} \chi_{(-r, r)}(x)}{\pi\left(1-x^{2}\right) \sqrt{r^{2}-x^{2}}}\left\{1-\left(\left|q_{L}\right|^{2}-\left|q_{R}\right|^{2}+\frac{a q_{L} \overline{b q_{R}}+\overline{a q_{L}} b q_{R}}{|a|^{2}}\right) x\right\},
$$

\footnotetext{
${ }^{2}$ Here, we listed up the experimental realizations of the DTQW. Therefore, Ref. 60] to experimentally demonstrate the two-particle continuous time quantum walk is omitted.

${ }^{3}$ The moment-generating function of the random variable under the specific time scale can be converged.
} 
where the indicator function $\chi_{(-r, r)}(x)$ is defined as

$$
\chi_{A}(x)=\left\{\begin{array}{ll}
1 & (x \in A) \\
0 & (x \notin A)
\end{array} .\right.
$$

While Konno used the combinatorial method in this proof [38, 39, in the following, we prove this by the spatial Fourier transformation [24].

Proof. Taking the Fourier transform for the state $|n, \xi\rangle$ with the position $n \in \mathbb{Z}$ and the coin state $|\xi\rangle \in \mathcal{H}_{c}$, we have

$$
|k, \hat{\xi}\rangle=\sum_{n \in \mathbb{Z}} e^{-i k n}|n, \xi\rangle .
$$

Since the time evolution in the phase space is given by

$$
\begin{aligned}
\left|\hat{\Psi}(k)_{t}\right\rangle & :=\sum_{n \in \mathbb{Z}} e^{-i k n} U^{t}\left|0, \phi_{0}\right\rangle \\
= & e^{i k}\left[\begin{array}{ll}
a & b \\
0 & 0
\end{array}\right] \sum_{n \in \mathbb{Z}} e^{-i k(n+1)}\left\langle n+1\left|U^{t-1}\right| 0, \phi_{0}\right\rangle \\
& +e^{-i k}\left[\begin{array}{cc}
0 & 0 \\
c & d
\end{array}\right] \sum_{n \in \mathbb{Z}} e^{-i k(n-1)}\left\langle n-1\left|U^{t-1}\right| 0, \phi_{0}\right\rangle \\
= & {\left[\begin{array}{cc}
e^{i k} & 0 \\
0 & e^{-i k}
\end{array}\right] U\left|\hat{\Psi}(k)_{t-1}\right\rangle }
\end{aligned}
$$

and the initial state in the phase space is given by

$$
\left|\hat{\Psi}(k)_{0}\right\rangle=q_{L}|k, \hat{L}\rangle+q_{R}|k, \hat{R}\rangle,
$$

we obtain

$$
\left|\hat{\Psi}(k)_{t}\right\rangle=U(k)^{t}\left|\hat{\Psi}(k)_{0}\right\rangle
$$

where

$$
U(k):=\left[\begin{array}{cc}
e^{i k} & 0 \\
0 & e^{-i k}
\end{array}\right] U .
$$

Here, we obtain the probability distribution in the real space,

$$
\operatorname{Pr}[n ; t]=\int_{-\pi}^{\pi} \frac{d k^{\prime}}{2 \pi} \int_{-\pi}^{\pi} \frac{d k}{2 \pi} e^{i\left(k-k^{\prime}\right) n}\left\langle\hat{\Psi}\left(k^{\prime}\right)_{0}\left|U^{\dagger}\left(k^{\prime}\right)^{t} U(k)^{t}\right| \hat{\Psi}(k)_{0}\right\rangle .
$$

Therefore, the $j$-th moment for the random variable $X_{t}$ is given by

$$
\begin{aligned}
\operatorname{Ex}\left[\left(X_{t}\right)^{j}\right] & :=\sum_{n \in \mathbb{Z}} n^{j} \operatorname{Pr}\left[X_{t}=n\right] \\
& =\sum_{n \in \mathbb{Z}} \int_{-\pi}^{\pi} \frac{d k^{\prime}}{2 \pi} e^{-i k^{\prime} n}\left\langle\hat{\Psi}\left(k^{\prime}\right)_{t}\left|\int_{-\pi}^{\pi} \frac{d k}{2 \pi}\left\{\left(-i \frac{d}{d k}\right)^{j} e^{i k^{\prime} n}\right\}\right| \hat{\Psi}(k)_{t}\right\rangle \\
& =\int_{-\pi}^{\pi} \frac{d k}{2 \pi}\left\langle\hat{\Psi}(k)_{t}\left|\left(i \frac{d}{d k}\right)^{j}\right| \hat{\Psi}(k)_{t}\right\rangle .
\end{aligned}
$$


Here, in the last line, we take the partial integration and use the periodic function for $\left|\hat{\Psi}(k)_{t}\right\rangle$.

Let the eigenvalue and its eigenvector of $U(k)$ be denoted as $\lambda_{\xi}(k)$ and $\left|v_{\xi}(k)\right\rangle$ with $\xi \in\{1,2\}$, respectively. We have

$$
\left(i \frac{d}{d k}\right)^{j}\left|\hat{\Psi}(k)_{t}\right\rangle=\sum_{\xi \in\{1,2\}} t(t-1) \cdots(t-j+1) \lambda_{\xi}(k)^{t-j}\left\langle v_{\xi}(k) \mid \hat{\Psi}(k)_{0}\right\rangle\left|v_{\xi}(k)\right\rangle+O\left(t^{j-1}\right) .
$$

Therefore, Eq. (16) is expressed as

$$
\begin{aligned}
\operatorname{Ex}\left[\left(X_{t}\right)^{j}\right]= & \int_{-\pi}^{\pi} \sum_{\xi \in\{1,2\}} t(t-1) \cdots(t-j+1) \lambda_{\xi}(k)^{-j}\left(i \frac{d}{d k} \lambda_{\xi}(k)\right)^{j} \times \\
& \left\langle v_{\xi}(k) \mid \hat{\Psi}(k)_{0}\right\rangle\left\langle\hat{\Psi}(k)_{0} \mid v_{\xi}(k)\right\rangle+O\left(t^{-1}\right) \\
= & \int_{-\pi}^{\pi} \sum_{\xi \in\{1,2\}} t(t-1) \cdots(t-j+1)\left(\frac{i \frac{d}{d k} \lambda_{\xi}(k)}{\lambda_{\xi}(k)}\right)^{j}\left|\left\langle v_{\xi}(k) \mid \hat{\Psi}(k)_{0}\right\rangle\right|^{2} \frac{d k}{2 \pi}+O\left(t^{-1}\right) .
\end{aligned}
$$

Therefore, we obtain, as $t \rightarrow \infty$,

$$
\operatorname{Ex}\left[\left(\frac{X_{t}}{t}\right)^{j}\right] \rightarrow \int_{-\pi}^{\pi} \sum_{\xi \in\{1,2\}}\left(\frac{i \frac{d}{d k} \lambda_{\xi}(k)}{\lambda_{\xi}(k)}\right)^{j}\left|\left\langle v_{\xi}(k) \mid \hat{\Psi}(k)_{0}\right\rangle\right|^{2} \frac{d k}{2 \pi}
$$

to obtain the momentum-generating function as

$$
\operatorname{Ex}\left[e^{\left(i \tau X_{t}\right) / t}\right] \rightarrow \sum_{\xi \in\{1,2\}} \int_{-\pi}^{\pi} \exp \left(i \tau \frac{i \frac{d}{d k} \lambda_{\xi}(k)}{\lambda_{\xi}(k)}\right)\left|\left\langle v_{\xi}(k) \mid \hat{\Psi}(k)_{0}\right\rangle\right|^{2} \frac{d k}{2 \pi} \text { as } t \rightarrow \infty
$$

for any real parameter $\tau \in \mathbb{R}$ since $\exp (i \tau f(k))$ is regular when $f(k)$ is a continuous function. We calculate the eigenvalue of Eq. (14) to obtain the term of $\frac{i \frac{d}{d k} \lambda_{\xi}(k)}{\lambda_{\xi}(k)}$ as

$$
\frac{i \frac{d}{d k} \lambda_{\xi}(k)}{\lambda_{\xi}(k)}= \pm \frac{|a| \cos \left(k-\frac{\theta}{2}\right)}{\sqrt{|a|^{2} \cos ^{2}\left(k-\frac{\theta}{2}\right)-\left(1-|a|^{2}\right) e^{i 2 k}}}=: \pm x
$$

where $e^{i \theta}:=a d-b c$ due to the unitary condition $|a d-b c|=1$. From Eq. (20) and $\left|\left\langle v_{1}(k) \mid \hat{\Psi}(k)_{0}\right\rangle\right|^{2}+\left|\left\langle v_{2}(k) \mid \hat{\Psi}(k)_{0}\right\rangle\right|^{2}=1$, we obtain

$$
\operatorname{Pr}\left[X_{t} \leq x\right]=\int_{-k(x)}^{k(x)}\left|\left\langle v_{1}(k) \mid \hat{\Psi}(k)_{0}\right\rangle\right|^{2} \frac{d k}{2 \pi}+\left(\int_{-\pi}^{-\pi+k(x)}+\int_{\pi-k(x)}^{\pi}\right)\left|\left\langle v_{2}(k) \mid \hat{\Psi}(k)_{0}\right\rangle\right|^{2} \frac{d k}{2 \pi}
$$


Therefore, we obtain the probability density as

$$
\begin{aligned}
f(x)= & \frac{d}{d x} \operatorname{Pr}\left[X_{t} \leq x\right] \\
= & \frac{1}{2 \pi}\left(\left|\left\langle v_{1}(k) \mid \hat{\Psi}(k)_{0}\right\rangle\right|^{2}+\left|\left\langle v_{1}(-k) \mid \hat{\Psi}(-k)_{0}\right\rangle\right|^{2}\right. \\
& \left.\quad \quad+\left|\left\langle v_{2}(-\pi+k) \mid \hat{\Psi}(-\pi+k)_{0}\right\rangle\right|^{2}+\left|\left\langle v_{2}(\pi-k) \mid \hat{\Psi}(\pi-k)_{0}\right\rangle\right|^{2}\right) \frac{d k(x)}{d x} \\
= & \frac{\sqrt{1-|a|^{2}} \chi_{(-|a|,|a|)}(x)}{\pi\left(1-x^{2}\right) \sqrt{|a|^{2}-x^{2}}}\left\{1-\left(\left|q_{L}\right|^{2}-\left|q_{R}\right|^{2}+\frac{a q_{L} \overline{b q_{R}}+\overline{a q_{L}} b q_{R}}{|a|^{2}}\right) x\right\} .
\end{aligned}
$$

This is the desired result.

The above theorem tells us that the DTQW has the capacities of the quantum speedup since the scaling of the random variable of the DTQW is $t$ while one of the classical case is $\sqrt{t}$. This effect originates from the superposition of the quantum walker. Also, the effect of the quantum interference causes a inverted bell shape distribution, which is completely different from the normal distribution.

\section{Connection to Dirac Equation}

In the following, we derive the free Dirac equation from the DTQW with the specified coin operator;

$$
C(\epsilon)=\left(\begin{array}{cc}
\cos \epsilon & -i \sin \epsilon \\
-i \sin \epsilon & \cos \epsilon
\end{array}\right)
$$

with $\epsilon \in \mathbb{R}$. Let $\Psi_{n}(x) \in \mathcal{H}_{p} \otimes \mathcal{H}_{c}$ be a quantum state at a position $x \in \mathbb{R}$ with the associated coin state $\left|\psi_{x}\right\rangle$ at $n$ step. When we take the tiny parameter $\epsilon$ as one lattice size, we obtain the dynamics of the DTQW as

$$
\begin{aligned}
\Psi_{n}(x) & =Q(\epsilon) \Psi_{n-1}(x-\epsilon)+P(\epsilon) \Psi_{n-1}(x+\epsilon) \\
& =Q(\epsilon)\left(1-\epsilon \frac{\partial}{\partial x}+O\left(\epsilon^{2}\right)\right) \Psi_{n-1}(x)+P(\epsilon)\left(1+\epsilon \frac{\partial}{\partial x}+O\left(\epsilon^{2}\right)\right) \Psi_{n-1}(x)
\end{aligned}
$$

where

$$
\begin{aligned}
& Q(\epsilon)=\left(\begin{array}{ll}
0 & 0 \\
0 & 1
\end{array}\right)-i \epsilon\left(\begin{array}{ll}
0 & 0 \\
1 & 0
\end{array}\right)+O\left(\epsilon^{2}\right) \\
& P(\epsilon)=\left(\begin{array}{ll}
1 & 0 \\
0 & 0
\end{array}\right)-i \epsilon\left(\begin{array}{ll}
0 & 1 \\
0 & 0
\end{array}\right)+O\left(\epsilon^{2}\right) .
\end{aligned}
$$

We obtain the dynamics of the DTQW from the beginning [6,77,

$$
\Psi_{\tau}(x) \sim e^{-i\left(\sigma_{x}+\sigma_{z} \frac{\partial}{\partial x}\right) t} \Psi_{0}(x),
$$


where $t=\epsilon \tau$. Partially differentiating this with respect to $t$, we obtain

$$
\begin{aligned}
\frac{\partial \Psi_{t}(x)}{\partial t} & \sim-i\left(\sigma_{x}+\sigma_{z} \frac{\partial}{\partial x}\right) e^{-i\left(\sigma_{x}+\sigma_{z} \frac{\partial}{\partial x}\right) t} \Psi_{0}(x) \\
& =-i\left(\sigma_{x}+\sigma_{z} \frac{\partial}{\partial x}\right) \Psi_{t}(x) .
\end{aligned}
$$

This equation corresponds to the Dirac equation in $1+1$ dimensions by taking the coin state as the spinor 4 . It is emphasized that the DTQW is described in non-relativistic quantum mechanics and the Dirac equation is the fundamental equation of relativistic quantum mechanics. This extension originates from the discretization of non-relativistic quantum mechanics. As a naive extension, some kinds of differential equations may be simulated from the DTQW model.

\section{Connection to Continuous Time Quantum Walk}

In this section, we will see the relationship between the continuous time quantum walk (CTQW), which will be defined in the following subsection, and the DTQW according to Ref. [17.

\subsection{Review of continuous time quantum walk}

Let us define the CTQW [19], which was originally motivated in the context of the speedup algorithm of quantum computation using the graph, as follows. The state space is defined as the position state space $\mathcal{H}_{p}$ only in contrast with the DTQW. Let $\Psi_{t}^{(C)}(x)$ be a quantum state at a position $x \in \mathbb{R}$. The time evolution is given by the discretized Schrödinger equation:

$$
\begin{aligned}
-i \frac{\partial \Psi_{t}^{(C)}(x)}{\partial t} & =\frac{1}{2}\left(\gamma \Psi_{t}^{(C)}(x-1)+\bar{\gamma} \Psi_{t}^{(C)}(x+1)\right) \quad \text { where } t>0, \\
\Psi_{0}^{(C)}(x) & =\delta(x),
\end{aligned}
$$

where $\gamma$ is a complex number. Let $X_{t}^{(C)}$ be the random variable of the CTQW at time $t$. The distribution of $X_{t}^{(C)}$ is given by $\operatorname{Pr}\left(X_{t}^{(C)}=x\right)=\left|\Psi_{t}^{(C)}(x)\right|^{2}$. This can be taken as dynamics of the discretized Schrödinger equation. Furthermore, from the viewpoint of statistical physics, this can be taken as the hopping dynamics of the Hubbard model noting that the CTQW describes the single-particle behavior.

In analogy to the DTQW (7), we obtain the weak limit theorem as

Theorem 5.1 (Konno [40]). Let $X_{t}^{(C)}$ be the CTQW at time $t$.

$$
\frac{X_{t}^{(C)}}{t} \Rightarrow Z(\gamma)
$$

\footnotetext{
${ }^{4}$ This analysis can be extended to the two-dimensional space [66].
} 
Here, $Z(a)$ has the probability density;

$$
f(x ; a)=\frac{\chi_{(-|a|,|a|)}(x)}{\pi \sqrt{|a|^{2}-x^{2}}} .
$$

This limit distribution (32) is called the arcsine distribution since the cumulative distribution function is given by

$$
\operatorname{Pr}[Z(\gamma) \leq x]:=\int_{-\infty}^{x} f(y ; \gamma) d y= \begin{cases}0 & \text { on } x \leq-|\gamma| \\ \frac{1}{\pi} \arcsin \frac{x}{|\gamma|}+\frac{1}{2} & \text { on }-|\gamma|<x<|\gamma| \\ 1 & \text { on }|\gamma| \leq 1\end{cases}
$$

It is curiously remarked that the arcsine distribution (32) can be derived as the limit distribution of the quantum probability theory with the monotone independence [26] while the CTQW is independent of the stochastic process in quantum probability theory.

Proof. Taking the spatial Fourier transform

$$
\hat{\Psi}_{t}(k):=\sum_{x \in \mathbb{Z}} \Psi_{t}(x) e^{i k x},
$$

we obtain the solution of Eq. (30);

$$
-i \frac{\hat{\Psi}_{t}(k)}{\partial t}=\frac{1}{2}\left(\gamma e^{i k} \hat{\Psi}_{t}(k)+\bar{\gamma} e^{-i k} \hat{\Psi}_{t}(k)\right) ;
$$

as

$$
\hat{\Psi}_{t}(k)=\operatorname{Exp}[i|\gamma| t \cos (k+\arg (\gamma))] .
$$

From the definition of the spatial Fourier transform, the moment-generating function can be expressed as

$$
\begin{aligned}
\operatorname{Ex}\left[e^{i \tau X_{t}^{(C)} / t}\right] & =\int_{0}^{2 \pi} \hat{\Psi}_{t}^{\dagger}(k) \cdot \hat{\Psi}_{t}\left(k+\frac{\tau}{t}\right) \frac{d k}{2 \pi} \\
& =\int_{0}^{2 \pi} \operatorname{Exp}\left[i|\gamma| t\left\{\cos \left(k+\arg (\gamma)+\frac{\tau}{t}\right)-\cos (k+\arg (\gamma))\right\}\right] \frac{d k}{2 \pi} \\
& =\int_{0}^{2 \pi} \operatorname{Exp}\left[i|\gamma| t \sin (k+\arg (\gamma)) \frac{\tau}{t}+O\left(\frac{\tau^{2}}{t^{2}}\right)\right] \frac{d k}{2 \pi} \\
& \rightarrow \int_{0}^{2 \pi} \operatorname{Exp}[i|\gamma| \tau \sin (k+\arg (\gamma))] \frac{d k}{2 \pi} \text { as } t \rightarrow \infty \\
& =\int_{-|\gamma|}^{|\gamma|} \frac{\operatorname{Exp}[i \tau x]}{\pi \sqrt{|\gamma|^{2}-x^{2}}} d x \\
& =\int_{-\infty}^{\infty} e^{i \tau x} \frac{\chi_{(-|\gamma|,|\gamma|)}(x)}{\pi \sqrt{|\gamma|^{2}-x^{2}}} d x
\end{aligned}
$$


Here, in the last second line, we take the transformed variable $x:=|\gamma| \sin (k+$ $\arg (\gamma))$. On the range of the integration, since the parameter $k$ is ranged from 0 to $2 \pi$, the maximum and minimum values of the parameter $x$ are $|\gamma|$ and $-|\gamma|$, respectively. Since the parameter $x$ is doubly counted, we have the last second line multiplied by two. Therefore, we obtain the desired result.

\subsection{Final-time dependent random walk and lazy random walk}

To pursue the aim of this section, we introduce a final-time-dependent (FTD) walks, which are modified walks initiated by Strauch [76. Let $\tilde{t}$ be the final time, that is, a particle keeps walking until the final time comes. At first, we show a construction of the CTRW from FTD-DTRWs. Secondly, we give CTQWs in some limit of the FTD-DTQW which is a quantum analogue of the final time dependent RW. Here, we assume the parameter $r(\tilde{t})>0$ with $r(\tilde{t}) \rightarrow 0$ as $\tilde{t} \rightarrow \infty$ for the FTD-DTRW and FTD-DTQW.

Let us define the FTD-DTRW on $\mathbb{Z}$, where the "final time" means the time that a particle stops the walk. The average of the waiting time of particle movement is $1 / r(\tilde{t})$. Here, $r(\tilde{t})$ is the probability that the particle moves by the final time $\tilde{t}$. The number of particle movements in the walk by the final time $\tilde{t}$ is evaluated as $r(\tilde{t}) \tilde{t}$. A particle spends her most time being lazy since the rate of movements $r(\tilde{t})$ tends to 0 as $\tilde{t} \rightarrow \infty$. This is called a lazy RW. In the following, we will show that the lazy RW with the number of particle's movements $\tilde{\operatorname{tr}} r(\tilde{t})$ can be taken as the CTRW with the final time $t$ for sufficiently large $\tilde{t}$.

Let $Z_{m}^{(F)}$ be the lazy RW at time $m \in\{0,1,2, \ldots, \tilde{t}\}$ defined by

$p_{m}(x)=(1-r(\tilde{t})) p_{m-1}(x)+\frac{r(\tilde{t})}{2}\left\{p_{m-1}(x-1)+p_{m-1}(x+1)\right\}, p_{0}(x)=\delta_{x, 0}$,

where $p_{m}(x):=\operatorname{Pr}\left(Z_{m}^{(F)}=x\right)(x \in \mathbb{N})$. This means that a particle stays at the same place with the probability $1-r(\tilde{t})$, and the particle jumps left or right with probability $1 / 2$ when the moving opportunity comes with probability $r(\tilde{t})$. Put a vector of the probability density as

$$
\left|\mu_{m}\right\rangle:=\left[\begin{array}{c}
\vdots \\
p_{m}(-1) \\
p_{m}(0) \\
p_{m}(1) \\
\vdots
\end{array}\right] \in \mathcal{H}_{p}
$$

It is noted that this vector $\left|\mu_{m}\right\rangle$ can be expressed as the real-valued vector. Equation (38) is equivalent to

$$
\left|\mu_{m}\right\rangle=\left\{\mathbf{1}+r(\tilde{t})\left(\frac{A}{2}-\mathbf{1}\right)\right\}\left|\mu_{m-1}\right\rangle,\left|\mu_{0}\right\rangle=|0\rangle,
$$


where $A|x\rangle:=|x+1\rangle+|x-1\rangle$ for any $x \in \mathbb{N}$. Here, $\left|\mu_{0}\right\rangle$ means $p_{0}(x)=\delta_{x, 0}$. Therefore, we have under the assumption of $\tilde{t} r^{2}(\tilde{t}) \rightarrow 0$ as $\tilde{t} \rightarrow \infty$,

$$
\left|\mu_{\tilde{t}}\right\rangle \sim \operatorname{Exp}\left[\tilde{t r}(\tilde{t})\left(\frac{A}{2}-\mathbf{1}\right)\right]\left|\mu_{0}\right\rangle
$$

Replace the particle movements $\tilde{t} r(\tilde{t})$ with a continuous parameter $t$. Partially differentiating Eq. (41) with respect to the parameter $t$, we obtain

$$
\begin{aligned}
\frac{\partial}{\partial t}\left|\mu_{t}\right\rangle & \sim\left(\frac{A}{2}-\mathbf{1}\right) \operatorname{Exp}\left[t\left(\frac{A}{2}-\mathbf{1}\right)\right]\left|\mu_{0}\right\rangle \\
& \sim\left(\frac{A}{2}-\mathbf{1}\right)\left|\mu_{t}\right\rangle .
\end{aligned}
$$

Therefore, we can express $p_{\tilde{t}}(x) \sim m_{s}(x)$ for sufficiently large $\tilde{t}$, where $m_{s}(x)$ satisfies

$$
\frac{\partial}{\partial s} m_{s}(x)=\frac{1}{2}\left\{m_{s}(x+1)+m_{s}(x-1)\right\}-m_{s}(x), m_{0}(x)=\delta(x) \text { on } s \leq t .
$$

This differential equation corresponds to the CTRW with time $t$. We have the central limit theorem by using the Fourier transform in the following: if $\tilde{t} r(\tilde{t}) \rightarrow \infty$, as $\tilde{t} \rightarrow \infty$, then

$$
\frac{Z_{\tilde{t}}^{(F)}}{\sqrt{\tilde{t} r(\tilde{t})}} \Rightarrow N(0,1) \text { as } \tilde{t} \rightarrow \infty .
$$

It is remarked that this equation can be shown without $\tilde{t} r(\tilde{t}) \rightarrow 0$ as $\tilde{t} \rightarrow \infty$ In particular, in the case of $r(\tilde{t}) \sim r / \tilde{t}^{\alpha}$ with $0<r<1$ and $0 \leq \alpha$, we obtain a crossover from the diffusive to the localized spreading: as $\tilde{t} \rightarrow \infty$, if $0 \leq \alpha<1$, then

and if $\alpha=1$, then

$$
\frac{Z_{\tilde{t}}^{(F)}}{\sqrt{\tilde{t}^{1-\alpha}}} \Rightarrow N(0, r)
$$

$$
\operatorname{Pr}\left(Z_{\tilde{t}}^{(F)}=n\right) \sim e^{-r} I_{n}(r),
$$

where $I_{\nu}(z)$ is the modified Bessel function of order $\nu$;

$$
I_{\nu}(z)=\sum_{m=0}^{\infty} \frac{1}{2^{2 m+\nu} \Gamma(m+1) \Gamma(\nu+m+1)} z^{2 m+\nu} .
$$

Here, the gamma function $\Gamma(z)$ is given by

$$
\Gamma(z)=\int_{0}^{\infty} y^{1-z} e^{-t} d y .
$$

Note that Eq.(46) comes from the correspondence between $\tilde{t} r(\tilde{t})$ and $t$, and the Fourier transform for Eq. (43): for large $\tilde{t}$,

$$
p_{\tilde{t}}(x) \sim m_{t}(x)=e^{-t} I_{x}(t),
$$

where and if $\alpha>1$, then we can easily see that $\operatorname{Pr}\left(Z_{\tilde{t}}^{(F)}=x\right) \rightarrow \delta(x)$. 


\subsection{Final-time dependent discrete time quantum walk}

In the following, we will consider a quantum-mechanical analogue of the above method of continuum approximation to the lazy RW. The FTD coin operator is defined by

$$
C_{\tilde{t}}:=\left[\begin{array}{cc}
\sqrt{r(\tilde{t})} & \sqrt{1-r(\tilde{t})} \\
\sqrt{1-r(\tilde{t})} & -\sqrt{r(\tilde{t})}
\end{array}\right] .
$$

This is defined as a quantum-mechanical analogue to the FTD stochastic coin of the correlated RW defined [42. According to Ref. [76], the absolute values of diagonal parts of the quantum coin are sufficiently small and independent of the final time $\tilde{t}$. According to Ref. 64, the coin operator is changed at each time, and its diagonal parts at time $m(<\tilde{t})$ are given in proportion to $1 / m^{\alpha}$ $(0 \leq \alpha \leq 1)$. On the other hand, in our model, all elements of the quantum coin depend on the final time $\tilde{t}$. The above quantum coin (50) shows that a particle moves in the same direction of the previous step with the probability amplitude $\sqrt{r(\tilde{t})}$ (left case) and $-\sqrt{r(\tilde{t})}$ (right case), and the opposite one with $\sqrt{1-r(\tilde{t})}$ (left and right cases). This is called an FTD-DTQW. We give the following lemma which shows that the FTD-DTQW is expressed as a linear combination of some CTQWs for sufficiently large $\tilde{t}$. The following lemma is consistent to Refs. 64,76] except for the time scaling. Let $\tilde{t}$ be the final time for the FTD-DTQW and $t$ be the time for the corresponding CTQWs. We define a quantum analogue of the waiting time of a quantum particle movement by $\tilde{t} / t$.

Lemma 5.2. Let $\Psi_{n}^{(F)}(x)$ be the coin state of the FTD-DTQW at time $\tilde{t}$ and position $x$. Put $t=\tilde{t} \sqrt{r(\tilde{t})}$ with $\tilde{t} r(\tilde{t})=o(1)$ for large $\tilde{t} . \Psi_{\tilde{t}}^{(F)}(x)$ is asymptotically expressed by

$$
\Psi_{\tilde{t}}^{(F)}(x) \sim \frac{1}{2}\left(\Psi_{t}^{(+)}(x)+(-1)^{\tilde{t}} \Psi_{t}^{(-)}(x)\right)
$$

with $t=n \sqrt{r(\tilde{t})}$, where $\Psi_{s}^{( \pm)}(x)$ satisfies the following Schrödinger equation:

$$
\begin{aligned}
-i \frac{\partial}{\partial s} \Psi_{s}^{( \pm, \xi)}(x) & = \pm \frac{1}{2}\left(i \Psi_{s}^{( \pm, \xi)}(x-1)-i \Psi_{s}^{( \pm, \xi)}(x+1)\right), \quad(\xi \in\{L, R\}) \\
\Psi_{0}^{( \pm, L)}(x) & =q_{L} \delta_{x, 0} \pm q_{R} \delta_{x, 1}, \quad \Psi_{0}^{( \pm, R)}(x)=q_{R} \delta_{x, 0} \pm q_{L} \delta_{x,-1},
\end{aligned}
$$

where $\Psi_{s}^{( \pm, \xi)}(x)=\left\langle\xi \mid \Psi_{s}^{( \pm)}(x)\right\rangle,(\xi \in\{R, L\})$.

It is noted that Eq. (52) can be taken as the CTQW with $\gamma= \pm i$ and the modified initial condition Eq. (53).

Proof. By the spatial Fourier transform for Eq. (50), we obtain

$$
\widehat{C}_{\tilde{t}}^{2}(k)=\mathbf{1}-2 i \sqrt{r(\tilde{t})} \sin k V_{\sigma_{x}}(k)+O(r(n)),
$$

with $V_{\sigma_{x}}(k):=\left(e^{-i k}|L\rangle\left\langle L\left|+e^{i k}\right| R\right\rangle\langle R|\right) \sigma_{x}$. When $\left|\operatorname{det}\left(\sqrt{r(\tilde{t})} \sin k V_{\sigma_{x}}(k)\right)\right|<1$, so

$$
\log \left(\widehat{C}_{\tilde{t}}^{2}(k)\right)=-2 i \sqrt{r(\tilde{t})} \sin k V_{\sigma_{x}}(k)+O(r(\tilde{t})),
$$


where $\operatorname{det}(A)$ is the determinant of $A$. Therefore, we have under the assumption of $\tilde{t} r(\tilde{t}) \rightarrow 0$ as $\tilde{t} \rightarrow \infty$,

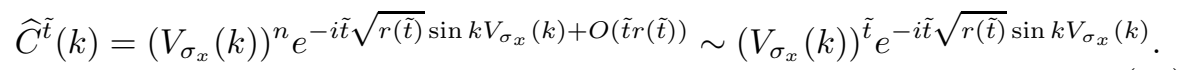

Because of $\left(\mathbf{1} \pm V_{\sigma_{x}}(k)\right) e^{-i s \sin k V_{\sigma_{x}}(k)}=e^{\mp i s \sin k}\left(\mathbf{1} \pm V_{\sigma_{x}}(k)\right)$ for any real number $s$, we obtain

$$
\widehat{C}^{\tilde{t}}(k) \sim \frac{1}{2}\left(e^{-i \tilde{t} \sqrt{r(\tilde{t})} \sin k}\left(\mathbf{1}+V_{\sigma_{x}}(k)\right)+(-1)^{\tilde{t}} e^{i \tilde{t} \sqrt{r(\tilde{t})} \sin k}\left(\mathbf{1}-V_{\sigma_{x}}(k)\right)\right) .
$$

To see a relationship between the FTD-DTQW and the CTQW, we define $\widehat{\Psi}_{s}^{( \pm)}(k) \equiv e^{\mp i s \sin k} \widehat{\Psi}_{0}^{( \pm)}(k)$ with $\widehat{\Psi}_{0}^{( \pm)}(k) \equiv\left(\mathbf{1} \pm V_{\sigma_{x}}(k)\right)\left|\phi_{0}\right\rangle$, where $\left|\phi_{0}\right\rangle$ is the initial coin state. It is noted that

$$
\widehat{\Psi}_{\tilde{t}}(k)=\frac{\widehat{\Psi}_{t}^{(+)}(k)+(-1)^{\tilde{t}} \widehat{\Psi}_{t}^{(-)}(k)}{2},
$$

with $t=\tilde{t} \sqrt{r(\tilde{t})}$ and $\widehat{\Psi}_{s}^{( \pm)}(k)$ obeys

$$
\pm i \frac{d}{d s} \widehat{\Psi}_{s}^{( \pm)}(k)=\sin k \widehat{\Psi}_{s}^{( \pm)}(k) .
$$

By the inverse Fourier transform for Eq. (57) and the definition of the Bessel function, we get the following theorem.

Theorem 5.3. Let $X_{\tilde{t}}^{(F)}$ be the FTD-DTQW at the final time $\tilde{t}$. Put $t=$ $\tilde{t} \sqrt{r(\tilde{t})}$. When the initial coin state is $\left|\phi_{0}\right\rangle=q_{L}|L\rangle+q_{R}|R\rangle$ with $\left|q_{L}\right|^{2}+\left|q_{R}\right|^{2}=1$, then we have

$$
\operatorname{Pr}\left(X_{\tilde{t}}^{(F)}=x\right) \sim \frac{1+(-1)^{\tilde{t}+x}}{2} \mathcal{J}(x ; t),
$$

where

$$
\mathcal{J}(x ; t):=\left\{1-\left(\overline{q_{R}} q_{L}+q_{R} \overline{q_{L}}\right) \frac{2 x}{t}\right\} J_{x}^{2}(t)+\left|q_{L}\right|^{2} J_{x-1}^{2}(t)+\left|q_{R}\right|^{2} J_{x+1}^{2}(t) .
$$

Here $J_{\nu}(z)$ is the Bessel function of the first kind of order $\nu$ as

$$
J_{\nu}(z)=\sum_{m=0}^{\infty} \frac{(-1)^{m}}{2^{2 m+\nu} \Gamma(m) \Gamma(m+\nu)} z^{2 m+\nu} .
$$

The following theorem shows that the weak convergence theorem of the FTD-DTQW also holds without the assumption of $\tilde{t} r(\tilde{t}) \rightarrow 0$ as $\tilde{t} \rightarrow \infty$.

Theorem 5.4. Let the initial coin state be $\left|\phi_{0}\right\rangle=q_{L}|L\rangle+q_{R}|R\rangle$ with $\left|q_{L}\right|^{2}+$ $\left|q_{R}\right|^{2}=1$. Assume $\tilde{t} \sqrt{r(\tilde{t})} \rightarrow \infty$ as $\tilde{t} \rightarrow \infty$. Then we have as $\tilde{t} \rightarrow \infty$,

$$
\frac{X_{\tilde{t}}^{(F)}}{\tilde{t} \sqrt{r(\tilde{t})}} \Rightarrow A^{\phi_{0}}(1) \text {, }
$$


where $A^{\phi_{0}}(y)$ has the following density:

$$
f(x ; y)=\frac{\chi_{(-|y|,|y|)}(x)}{\pi \sqrt{y^{2}-x^{2}}}\left\{1-\frac{\left(\overline{q_{R}} q_{L}+q_{R} \overline{q_{L}}\right) x}{y}\right\} .
$$

Proof. We show that Eq. (63) holds without assumption $\tilde{t} r(\tilde{t}) \rightarrow 0$ as $\tilde{t} \rightarrow \infty$. The Fourier transform for the quantum coin $C_{\tilde{t}}$ (50) is described as

$$
\widehat{C}_{\tilde{t}}(k)=\left[\begin{array}{cc}
e^{-i k} \sqrt{r(\tilde{t})} & e^{-i k} \sqrt{1-r(\tilde{t})} \\
e^{i k} \sqrt{1-r(\tilde{t})} & -e^{i k} \sqrt{r(\tilde{t})}
\end{array}\right] .
$$

Let the eigenvalue and corresponding eigenvector of $\widehat{C}_{\tilde{t}}(k)$ be $e^{i \theta_{\tilde{t}}^{( \pm)}(k)}$ and $\left|v_{\tilde{t}}^{( \pm)}(k)\right\rangle$. Then we obtain

$$
\begin{aligned}
\cos \theta_{n}^{( \pm)}(k) & = \pm \sqrt{1-r(\tilde{t}) \sin ^{2} k} \\
\sin \theta_{\tilde{t}}^{( \pm)}(k) & =-\sqrt{r(\tilde{t})} \sin k \\
\pi_{\tilde{t}}^{( \pm)}(k) \equiv\left|v_{\tilde{t}}^{( \pm)}(k)\right\rangle v_{\tilde{t}}^{( \pm)}(k) \mid & =\frac{1}{2}\left(\mathbf{1} \pm V_{\sigma_{x}}(k)\right)+O(\sqrt{r(\tilde{t})}) .
\end{aligned}
$$

Let $X_{s}^{(F)}$ be the FTD-DTQW at time $s$ with the initial coin state $\left|\phi_{0}\right\rangle=q_{L}|L\rangle+$ $q_{R}|R\rangle$. It is noted that

$$
\theta_{\tilde{t}}^{( \pm)}(k+\xi / t)-\theta_{\tilde{t}}^{( \pm)}(k)=\mp \xi \frac{\sqrt{r(\tilde{t})}}{t} \cos k+O\left(\frac{(\sqrt{r(\tilde{t})})^{3}}{t}\right),
$$

where $t=\tilde{t} \sqrt{r(\tilde{t})}$. We have

$$
\begin{aligned}
\operatorname{Ex}\left[e^{i \xi X_{\tilde{t}}^{(F)} / t}\right]= & \int_{0}^{2 \pi}\left\langle\phi_{0}\left|\left(\widehat{C}^{\tilde{t}}(k)\right)^{\dagger} \widehat{C}^{\tilde{t}}(k+\xi / t)\right| \phi_{0}\right\rangle \frac{d k}{2 \pi} \\
= & \int_{0}^{2 \pi} \sum_{j \in\{0,1\}} e^{-(-1)^{j} i \xi \cos k+O(\sqrt{r(\tilde{t})})} \\
& \times \frac{1}{2}\left\langle\phi_{0}\left|\left(\mathbf{1}+(-1)^{j} V_{\sigma_{x}}(k)\right)\right| \phi_{0}\right\rangle \frac{d k}{2 \pi}+O(\sqrt{r(\tilde{t})}) \\
\rightarrow & \int_{-\infty}^{\infty} e^{i \xi x}\left\{1-\left(q_{L} \overline{q_{R}}+\overline{q_{L}} q_{R}\right) x\right\} \frac{\chi_{(-1,1)}(x)}{\pi \sqrt{1-x^{2}}} d x \text { as } \tilde{t} \rightarrow \infty,
\end{aligned}
$$

where $t=\tilde{t} \sqrt{r(\tilde{t})}$. Then, we get the desired conclusion.

From Theorems 5.3 and 5.4, we obtain the following limit theorem which shows a crossover from the localized to the ballistic spreading.

Corollary 5.5. If $\sqrt{r(\tilde{t})}=r / \tilde{t}^{\alpha}$ with $0<r<1$, then as $\tilde{t} \rightarrow \infty$,

$$
\frac{X_{\tilde{t}}^{(F)}}{\tilde{t}^{1-\alpha}} \Rightarrow \begin{cases}K(r) & \text { if } \alpha=0, \\ A^{\phi_{0}}(r) & \text { if } 0<\alpha<1,\end{cases}
$$


and if $\alpha=1$, then

$$
\operatorname{Pr}\left(X_{\tilde{t}}^{(F)}=x\right) \sim \frac{1+(-1)^{n+x}}{2} \mathcal{J}(x ; r) .
$$

and if $\alpha>1$, then

$$
\operatorname{Pr}\left(X_{\tilde{t}}^{(F)}=x\right) \rightarrow \delta(x)
$$

as $\tilde{t} \rightarrow \infty$.

Proof. Since the $\alpha=0$ case corresponds to the DTQW, $X_{\tilde{t}}^{(F)} / \tilde{t} \Rightarrow K(r)$ as $\tilde{t} \rightarrow \infty$. The $\sqrt{r(\tilde{t})}=\tilde{t}^{\alpha}$ with $0<\alpha<1$ satisfies the condition $\tilde{t} \sqrt{r(n)} \rightarrow 0$ as $\tilde{t} \rightarrow \infty$. Therefore, it follows from Theorem $\left[5.4\right.$ that $X_{\tilde{t}}^{(F)} / \tilde{t}^{1-\alpha} \Rightarrow A^{\phi_{0}}$ with $0<\alpha<1$. The result on $\alpha \geq 1$ case derives from Theorem 5.3 .

\section{One-dimensional Discrete Time Quantum Walk as Quantum Simulator}

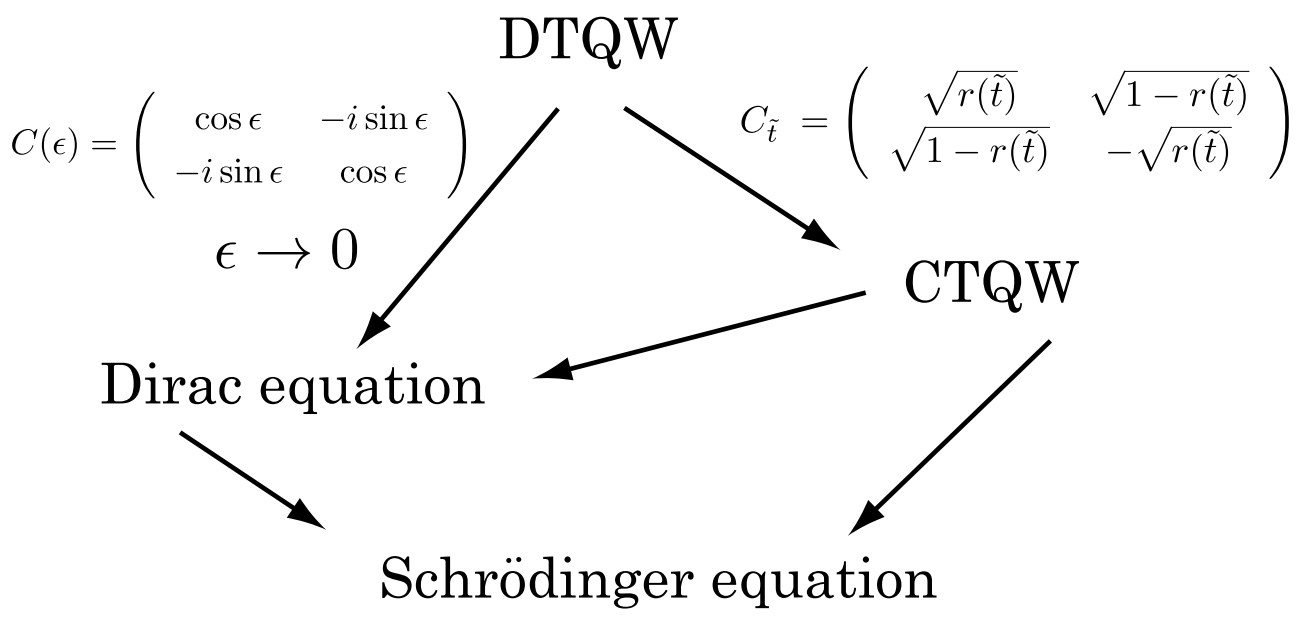

Figure 5: The relationship among the DTQW, the CTQW, and the Dirac and Schrödinger equations on a one-dimensional space for the asymptotic behaviors. The DTQW can derive the Dirac equation and the CTQW. These can derive the Schrödinger equation. It is noted that the CTQW on the graph can derive the $1+1$ dimensional Dirac equation [14].

Summarizing the above results, the DTQW can simulate the Dirac equation and the CTQW as the asymptotic behavior on the one-dimensional space. While the DTQW is run under non-relativistic quantum mechanics, the DTQW can simulate the relativistic situation. Therefore, the DTQW can be taken as a quantum simulator, which is different from the original idea of Richard Feynman [20]. Realizing the DTQW means that such experimental setup can test 
the quantum world. Compared to the original theory, the parameters of the quantum coin and the shift in the real space may be connected to the parameters and the constant number in the original theory. This means that we can construct the alternative theory beyond the quantum theory inside quantum theory. It should be emphasized that this theory is not realized but is consistent and can be interpreted. However, the DTQW has the potential to the test of the physical constant by changing this as the parameter in the alternative theory. On the other words in mathematics, the DTQW can derive the Dirac equation, the CTQW, and the Schrödinger equation. Here, the Schrödinger equation can be derived from the approximation of the Dirac equation and the CTQW. Therefore, the DTQW is mathematically a superordinate concept. These relationships can be illustrated in Fig. 5 .

\section{Acknowledgment}

The author acknowledges useful collaborations and discussion with Etsuo Segawa, Kota Chisaki and Norio Konno. The author also thanks Hosho Katsura and Takuya Kitagawa for useful discussion. The author would like to thank the use of the utilities of Tokyo Institute of Technology and Massachusetts Institute of Technology and many technical and secretary supports. The author is grateful to the financial supports from JSPS Research Fellowships for Young Scientists (No. 21008624), JSPS Excellent Young Researcher Overseas Visit Program, Global Center of Excellence Program "Nanoscience and Quantum Physics" at Tokyo Institute of Technology during his Ph.D study.

\section{A Classical stochastic process}

In this appendix, we define the stochastic process as

Definition A.1 (Stochastic process). The stochastic process is defined as

$$
\left\{X_{t}(\omega), t \in T\right\} \omega \in \Omega,
$$

where $T$ means time range and is assumed as the natural number $T=\mathbb{N}$ to simplify the discussion.

The simplest example is the Markov process, which is defined that $X_{t+1}$ is independent of $X_{t}$ for any $t \in T$. One of the Markov process is the random walk (RW).

Definition A.2 (Time-independent and spatial-independent one-dimensional random walk). The time-independent and spatial-independent one-dimensional random walk is defined as the independent and identical distributed (i.i.d.) sequence $X_{t}$ to satisfy

$$
\begin{aligned}
\operatorname{Pr}\left[X_{t+1}\right. & =x]=p \operatorname{Pr}\left[X_{t}=(x-1)\right]+(1-p) \operatorname{Pr}\left[X_{t}=(x+1)\right], \\
\operatorname{Pr}\left[X_{0}=x\right] & =\delta_{x, 0}
\end{aligned}
$$


for any $t \in \mathbb{N}$ and $x \in \mathbb{Z}$.

To describe the asymptotic behavior for the random variable $X_{n}$ with the suffix $n \in \mathbb{N}$, we define the weak convergence of distribution 5 as

Definition A.3 (Weak convergence of distribution). Let $\left\{X_{n}\right\}$ be the sequence of the random variables. The random variable $X_{n}$ weakly converges to the random variable $Y$ denoted as

$$
X_{n} \Rightarrow Y
$$

if and only if, for any $\epsilon>0$,

$$
\operatorname{Pr}\left[\left|X_{n}-Y\right|>\epsilon\right] \rightarrow 0 \text { as } n \rightarrow \infty .
$$

This definition can be expressed from the viewpoint of the probability measure as

$$
\lim _{n \rightarrow \infty} \int_{\omega \in \Omega} f\left(X_{n}[\omega]\right) d P_{n}[\omega]=\int_{\omega \in \Omega} f(Y[\omega]) d P[\omega],
$$

where $d P_{n}$ and $d P$ are defined in the random variable $X_{n}$ and $Y$, respectively. In the case of the time-independent and spatial-independent one-dimensional RW, we obtain the asymptotic behavior in the following:

Theorem A.1 (Central limit theorem 6). Let $\left\{Y_{n}\right\}_{n \in \mathbb{N}}$ be the i.i.d. sequence to satisfy

$$
\operatorname{Pr}\left[Y_{n}=1\right]=p \quad \operatorname{Pr}\left[Y_{n}=1\right]=1-p
$$

for any $n \in \mathbb{N}$. Put $S_{m}=\sum_{k=1}^{m} Y_{k}$. Then, we obtain

$$
\frac{S_{m}-\operatorname{Ex}\left[S_{m}\right]}{\sqrt{\operatorname{Var}\left[S_{m}\right]}} \Rightarrow N(0,1) \text { as } m \rightarrow \infty
$$

where $N(\mu, \nu)$ is the random variable to express the normal distribution 7 with the mean $\mu$ and the variance $\nu$ given by

$$
\operatorname{Pr}[N(\mu, \nu)=x]=\frac{1}{\sqrt{2 \pi \nu}} \operatorname{Exp}\left[-\frac{(x-\mu)^{2}}{2 \nu}\right] .
$$

Here, the expectation value and the variance are given by

$$
\begin{aligned}
\operatorname{Ex}\left[S_{m}\right] & =m p, \\
\operatorname{Var}\left[S_{m}\right] & =m p(1-p) .
\end{aligned}
$$

It is noted that the normal distribution (81) is characterized only by the mean and the variance.

\footnotetext{
${ }^{5}$ This is also called the weak convergence, convergence of distribution, or the probability convergence.

${ }^{6}$ This is often called the de Moivre-Laplace theorem. The general version of this theorem to satisfy Eq. (80) for the independent sequence $\left\{Y_{n}\right\}$ was proven by Lindeberg to see more details in the book 61 .

${ }^{7}$ In physics, this distribution is called the Gaussian.
} 
Proof. Given the function $T_{m}$ as

$$
T_{m}:=\sum_{k=1}^{m} \frac{Y_{k}-p}{\sqrt{m p(1-p)}},
$$

we obtain the moment-generating function for $T_{m}$ as

$$
\begin{aligned}
\operatorname{Ex}\left[e^{i \tau T_{m}}\right] & =\prod_{k=1}^{m} \operatorname{Ex}\left[\operatorname{Exp}\left(\frac{i \tau\left(Y_{k}-p\right)}{\sqrt{m p(1-p)}}\right)\right] \\
& =\left[p \operatorname{Exp}\left(i \tau \sqrt{\frac{(1-p)}{m p}}\right)+(1-p) \operatorname{Exp}\left(-i \tau \sqrt{\frac{p}{m(1-p)}}\right)\right]^{m} \\
& =\left[p\left(1+i \tau \sqrt{\frac{(1-p)}{m p}}-\frac{(1-p)}{2 m p} \tau^{2}\right)\right. \\
& \left.\quad+(1-p)\left(1-i \tau \sqrt{\frac{p}{m(1-p)}}-\frac{p}{2 m(1-p)} \tau^{2}\right)+O\left(\frac{1}{m^{2}}\right)\right]^{m} \\
= & {\left[1-\frac{\tau^{2}}{2 m}+O\left(\frac{1}{m^{2}}\right)\right]^{m} } \\
\rightarrow & \operatorname{Exp}\left(-\frac{\tau^{2}}{2}\right) \text { as } m \rightarrow \infty .
\end{aligned}
$$

From the inverse Fourier transform for the last line, we obtain the desired result.

Applying the central limit theorem to the time-independent and spatialindependent one-dimensional RW, we obtain the following corollary by replacing $S_{m}$ to $X_{t}$ :

Corollary A.2. The time-independent and spatial-independent one-dimensional $R W X_{t}$ has the asymptotic behavior as

$$
\frac{X_{t}}{\sqrt{t}} \Rightarrow N(2 p-1,4 p(1-p)) .
$$

Furthermore, in case of the unbiased coin, $p=1 / 2$, one has

$$
\frac{X_{t}}{\sqrt{t}} \Rightarrow N(0,1) \text {. }
$$

Proof. We calculate the expectation value of $X_{t}$ as

$$
\operatorname{Exp}\left[X_{t}\right]=\sum_{k=1}^{t}(p-(1-p))=t(2 p-1) .
$$

The variance is calculated as

$$
\operatorname{Var}\left[X_{t}\right]=\sum_{k=1}^{t}[p+(1-p)]-(2 p-1)^{2}=4 t p(1-p) .
$$

Applying them to Theorem A.1, we obtain the desired result. 


\section{References}

[1] G. Abal, R. Donangelo, and H. Fort, in Annals of the 1st Workshop on Quantum Computation and Information, p. 189, UCPel, 9-11 October. 2006, Pelotas, RS, Brazil, arXiv:0709.3279.

[2] Y. Aharonov, L. Davidovich, and N. Zagury, Phys. Rev. A 48, 1687 (1993).

[3] A. Ahlbrecht, A. Alberti, D. Meschede, V. B. Scholz, A. H. Werner, and R. F. Werner, arXiv:1105.1051.

[4] A. Ambainis, E. Bach, A. Nayak, A. Vishwanath, and J. Watrous, in Proceedings of the 33rd Annual ACM Symposium on Theory of Computing (STOC'01) (ACM Press, New York, 2001), p. 37.

[5] A. Ambainis, SIAM Journal on Computing 37, 210 (2007).

[6] A. J. Bracken, D. Ellinas, and I. Smyrnakis, Phys. Rev. A 75, 022322 (2007).

[7] M. A. Broome, A. Fedrizzi, B. P. Lanyon, I. Kassal, A. Aspuru-Guzik, and A. G. White, Phys. Rev. Lett. 104, 153602 (2010).

[8] H. Buhrman and R. Spalek, in Proc. 17th ACM-SIAM Symposium on Discrete Algorithms (Society for Industrial and Applied Mathematics, Philadelphia, 2006), p. 880.

[9] C. M. Chandrashekar, arXiv:quant-ph/0609113

[10] C. M. Chandrashekar, Cent. Eur. J. Phys., 8, 979 (2010).

[11] C. M. Chandrashekar, S. Banerjee, and R. Srikanth, Phys. Rev. A 81, 062340 (2010).

[12] C. M. Chandrashekar and R. Laflamme, Phys. Rev. A 78, 022314 (2008).

[13] A. M. Childs, Phys. Rev. Lett. 102, 180501 (2009).

[14] A. M. Childs and J. Goldstone, Phys. Rev. A 70, 042312 (2004).

[15] K. Chisaki, M. Hamada, N. Konno, and E. Segawa, Interdisciplinary Information Sciences 15, 423 (2009).

[16] K. Chisaki, N. Konno, and E. Segawa, Quant. Inf. Comp. 12, 0314 (2012).

[17] K. Chisaki, N. Konno, E. Segawa, and Y. Shikano, Quant. Inf. Comp. 11, 0741 (2011).

[18] K. Eckert, J. Mompart, G. Birkl, and M. Lewenstein, Phys. Rev. A 72, 012327 (2005).

[19] E. Farhi and S. Gutmann, Phys. Rev. A 58, 915 (1998). 
[20] R. P. Feynman, Int. J. Theor. Phys. 21, 467 (1982).

[21] P.-G. D. Gennes, Scaling Concepts in Polymer Physics (Cornell University Press, New York, 1979).

[22] R. Gerritsma, G. Kirchmair, F. Zähringer, E. Solano, R. Blatt, and C. F. Roos, Nature 463, 68 (2010).

[23] M. Gönülol, E. Aydıner, Y. Shikano, and Ö. E. Müstecaplıog̃lu, New J. Phys. 13, 033037 (2011).

[24] G. Grimmett, S. Janson, and P. F. Scudo, Phys. Rev. E. 69, 026119 (2004).

[25] S. P. Gudder, Quantum Probability (Academic Press Inc., San Diego, 1988).

[26] T. Hasebe, arXiv:1009.1505.

[27] N. Inui, Y. Konishi, and N. Konno, Phys. Rev. A 69, 052323 (2004).

[28] N. Inui, and N. Konno, Physica A 353, 133 (2005).

[29] N. Inui, N. Konno, and E. Segawa, Phys. Rev. E 72, 056112 (2005).

[30] A. Joye, Commun. Math. Phys. 307, 65 (2011).

[31] A. Joye and M. Merkli, J. Stat. Phys. 140, 1 (2010).

[32] M. Karski, L. Föster, J.-M. Choi, A. Steffen, W. Alt, D. Meschede, and A. Widera, Science 325, 174 (2009).

[33] J. Kempe, Contemp. Phys. 44, 307 (2003).

[34] V. Kendon, Math. Struct. in Comp. Sci. 17, 1169 (2007).

[35] T. Kitagawa, to be published from Quant. Inf. Proc., arXiv:1112.1882.

[36] T. Kitagawa, M. A. Broome, A. Fedrizzi, M. S. Rudner, E. Berg, I. Kassal, A. Aspuru-Guzik, E. Demler, and A. G. White, Nat. Comm. 3, 882 (2012).

[37] T. Kitagawa, M. S. Rudner, E. Berg, and E. Demler, Phys. Rev. A 82, 033429 (2010).

[38] N. Konno, Quantum Inf. Proc. 1, 345 (2002).

[39] N. Konno, J. Math. Soc. Jpn. 57, 1179 (2005).

[40] N. Konno, Phys. Rev. E 72, 026113 (2005).

[41] N. Konno, in Quantum Potential Theory, Lecture Notes in Mathematics Vol. 1954, edited by U. Franz and M. Schurmann (Springer-Verlag, Heidelberg, 2008), p.309. 
[42] N. Konno, Stochastic Models 25, 28 (2009).

[43] N. Konno, Quantum Inf. Proc. 8, 387 (2009).

[44] N. Konno, Quantum Inf. Proc. 9, 405 (2010).

[45] N. Konno and E. Segawa, Quant. Inf. Comp. 11, 0485 (2011).

[46] Y. Lahini, M. Verbin, S. D. Huber, Y. Bromberg, R. Pugatch, and Y. Silberberg, Phys. Rev. A 86, 011603 (2012).

[47] N. Linden and J. Sharam, Phys. Rev. A 80, 052327 (2009).

[48] C. Liu and N. Petulante, Phys. Rev. A 79, 032312 (2009).

[49] C. Liu and N. Petulante, Phys. Rev. A 84, 012317 (2011).

[50] N. B. Lovett, S. Cooper, M. Everitt, M. Trevers, and V. Kendon, Phys. Rev. A 81, 042330 (2010).

[51] T. D. Mackay, S. D. Bartlett, L. T. Stephenson, and B. C. Sanders, J. Phys. A 35, 2745 (2002).

[52] F. Magniez and A. Nayak, Algorithmica 48, 221 (2007).

[53] F. Magniez, M. Santha, and M. Szegedy, SIAM Journal of Computing 37, 413 (2007).

[54] O. Maloyer and V. Kendon, New J. Phys. 9, 87 (2007).

[55] K. Mayer, M. C. Tichy, F. Mintert, T. Konrad, and A. Buchleitner, Phys. Rev. A 83, 062307 (2011).

[56] M. McGettrick, Quant. Inf. Comp. 10, 0509 (2010).

[57] D. Meyer, J. Stat. Phys. 85, 551 (1996).

[58] H. Obuse and N. Kawakami, Phys. Rev. B 84, 195139 (2011).

[59] T. Oka, N. Konno, R. Arita, and H. Aoki, Phys. Rev. Lett. 94, 100602 (2005).

[60] A. Peruzzo, M. Lobino, J. C. F. Matthews, N. Matsuda, A. Politi, K. Poulios, X. Zhou, Y. Lahini, N. Ismail, K. Wörhoff, Y. Bromberg, Y. Silberberg, M. G. Thompson and J. L. O'Brien, Science 329, 1500 (2010).

[61] P. Revész, Random walk in random and non-random environments (World Scientific, Singapore, 1990).

[62] P. P. Rohde, A. Fedrizzi, and T. C. Ralph, J. Mod. Opt. 59, 710 (2012).

[63] A. Romanelli, Physica A 388, 3985 (2009). 
[64] A. Romanelli, Phys. Rev. A 80, 042332 (2009).

[65] C. A. Ryan, M. Laforest, J. C. Boileau, and R. Laflamme, Phys. Rev. A 72, 062317 (2005).

[66] F. Sato and M. Katori, Phys. Rev. A 81, 012314 (2010).

[67] A. Schreiber, K. N. Cassemiro, V. Potoček, A. Gábris, P. J. Mosley, E. Andersson, I. Jex, and Ch. Silberhorn, Phys. Rev. Lett. 104, 050502 (2010).

[68] N. Shenvi, J. Kempe, and K. Whaley, Phys. Rev. A 67, 052307, (2003).

[69] Y. Shikano, K. Chisaki, E. Segawa, and N. Konno, Phys. Rev. A 81, 062129 (2010).

[70] Y. Shikano and H. Katsura, Phys. Rev. E 82, 031122 (2010).

[71] Y. Shikano and H. Katsura, AIP Conf. Proc. 1363, 151 (2011).

[72] M. Štefaňák, S. M. Barnett, B. Kollár, T. Kiss, and I. Jex, New J. Phys. 13, 033029 (2011).

[73] M. Štefaňák, I. Jex, and T. Kiss, Phys. Rev. Lett. 100, 020501 (2008).

[74] M. Štefaňák, T. Kiss, and I. Jex, Phys. Rev. A 78, 032306 (2008).

[75] M. Štefaňák, T. Kiss, and I. Jex, New J. Phys. 11, 043027 (2009).

[76] F. W. Strauch, Phys. Rev. A 74, 030301 (2006).

[77] F. W. Strauch, J. Math. Phys. 48, 082102 (2007).

[78] B. Tregenna, W. Flanagan, R. Maile, and V. Kendon, New J. Phys. 5, 83 (2003).

[79] S. E. Venegas-Andraca, Quantum Walks for Computer Scientists (Morgan and Claypool, 2008).

[80] S. E. Venegas-Andraca, to be published from Quan. Inf. Proc., arXiv:1201.4780,

[81] K. Watabe, N. Kobayashi, M. Katori, and N. Konno, Phys. Rev. A 77, 062331 (2008).

[82] A. Wójcik, T. Łuczak, P. Kurzyński, A. Grudka, and M. Bednarska, Phys. Rev. Lett. 93, 180601 (2004).

[83] X.-P. Xu, Eur. Phys. J. B 77, 479 (2010).

[84] F. Zähringer, G. Kirchmair, R. Gerritsma, E. Solano, R. Blatt, and C. F. Roos, Phys. Rev. Lett. 104, 100503 (2010). 